\title{
A Novel Scheme for Material Updating in Source Distribution Optimization of Magnetic Devices Using Sensitivity Analysis
}

\author{
Dong-Hun Kim ${ }^{1}$, Jan K. Sykulski ${ }^{2}$, Senior Member, IEEE, and David A. Lowther ${ }^{3}$, Member, IEEE \\ ${ }^{1}$ School of Electrical Engineering and Computer Science, Kyungpook National University, Daegu 702-701, Korea \\ ${ }^{2}$ School of Electronics and Computer Science, Electrical Power \\ Engineering, University of Southampton, Southampton SO17 1BJ, U.K. \\ ${ }^{3}$ Department of Electrical and Computer Engineering, McGill University, Montreal, QC H3A 2A7 Canada
}

\begin{abstract}
A novel material updating scheme, which does not require intermediate states of a material used, is presented for source distribution optimization problems. A mutation factor to determine a degree of topological change in the next design stage on the basis of a current layout accelerates the convergence of an objective function. Easy implementation and fast convergence of the scheme are verified using two MRI design problems where current and permanent magnet distributions have been optimized, respectively.
\end{abstract}

Index Terms-Adjoint variable method, design sensitivity, topology optimization.

\section{INTRODUCTION}

$\mathbf{T}$ OPOLOGY OPTIMIZATION (TO), which originated in the structural mechanics community, has also been successfully applied to finding the optimized material distribution (OMD) and the optimized source distribution (OSD) in electromagnetic systems [1]-[3]. However, it is important to appreciate that there exists a difference between applications in electromagnetic and structural TO: In electromagnetic design, the OSD additionally deals with forcing terms of the system such as current density and permanent magnetization, unlike the structural TO. Moreover, from the point of view of the adjoint system of the sensitivity analysis, while the permeability of the primary system strongly distorts the adjoint field distribution, the magnetic sources do not affect the fields because they are replaced by air regions. This implies that the magnetic sources are very weakly coupled to the sensitivity coefficients, unlike the permeability. Consequently, a different approach —especially in the material updating scheme-should be taken when solving source distribution problems.

This paper presents a very fast and efficient TO algorithm for optimizing source distribution in linear magnetostatic problems. In order to make a distinction between OMD and OSD, a unified design sensitivity formula with respect to the system parameters of magnetic material and sources is first derived by exploiting the adjoint variable method (AVM) and the augmented Lagrangian method. Then, the effects of the magnetic properties on sensitivity coefficients are examined from the viewpoint of the mathematical expression and the construction of the adjoint system. This enables a novel material updating algorithm-which does not require intermediate states, nor any penalty functions of a material and an objective function used - to be applied to the OSD method. The scheme has been successfully combined with a commercial finite element code OPERA and the validity and efficiency of the approach

Digital Object Identifier 10.1109/TMAG.2005.846036 have been verified using two MRI design problems involving searching for optimized current and permanent magnet distributions, respectively.

\section{Derivation of a Unified Design Sensitivity}

The Tellegen's theorem, or mutual energy concept, in conjunction with AVM has been utilized to obtain analytical sensitivity formulae that contain the derivative information of an objective function to changes of material properties [1], [2]. However, it is recognized that the meaning and physical interpretation of the adjoint system in such formulations are often obscure and thus difficult to understand.

In this paper, by exploiting the AVM and the augmented objective function, a new approach to TO yielding a better understanding of the adjoint system is proposed. First, consider an objective function $F$, given by (1), which occurs when dealing with TO of magnetostatic systems

$$
F=\int_{\Omega} g(\mathbf{A}(\mathbf{p}), \operatorname{curl} \mathbf{A}(\mathbf{p})) d \Omega
$$

where $g$ is a scalar function differentiable with respect to the magnetic vector potential $\mathbf{A}$ and $\operatorname{curl} \mathbf{A}$, which are themselves implicit functions of the system parameter vector $\mathbf{p}$ : The permeability $\mu$, current density $\mathbf{J}$ and permanent magnetization $\mathbf{M}$. In order to deduce a design sensitivity formula and the adjoint system equation systematically, the variational of Maxwell's equation - referred to as the primary system - is added to (1) based on the augmented Lagrangian method

$$
\begin{aligned}
\bar{F}=\int_{\Omega} g(\mathbf{A}, \operatorname{curl} \mathbf{A}) d \Omega & \\
& +\int_{\Omega} \lambda[-\operatorname{curl}(v \operatorname{curl} \mathbf{A}-\mathbf{M})+\mathbf{J}] d \Omega
\end{aligned}
$$

where $v$ is the reluctivity and $\boldsymbol{\lambda}$ means the Lagrange multiplier vector interpreted as the adjoint variable. 
By taking the variation of both sides of (2) with respect to small changes $\delta \mathbf{p}$ of the system parameters, the augmented objective function can be developed as follows:

$$
\begin{aligned}
& \delta \bar{F}=\int_{\Omega}\left[\mathbf{g}_{\mathbf{A}} \cdot\left(\delta \mathbf{A}+\frac{\partial \mathbf{A}}{\partial \mathbf{p}} \delta \mathbf{p}\right)+\mathbf{g}_{\operatorname{curl} \mathbf{A}}\right. \\
& \left.\cdot \operatorname{curl}\left(\delta \mathbf{A}+\frac{\partial \mathbf{A}}{\partial \mathbf{p}} \delta \mathbf{p}\right)\right] d \Omega-\int_{\Omega}\left[v \operatorname{curl}\left(\delta \mathbf{A}+\frac{\partial \mathbf{A}}{\partial \mathbf{p}} \delta \mathbf{p}\right)\right. \\
& \left.\cdot \operatorname{curl} \boldsymbol{\lambda}+v \operatorname{curl} \mathbf{A} \cdot \operatorname{curl}\left(\delta \boldsymbol{\lambda}+\frac{\partial \boldsymbol{\lambda}}{\partial \mathbf{p}} \delta \mathbf{p}\right)\right] d \Omega \\
& +\int_{\Omega}\left[\mathbf{J} \cdot\left(\delta \boldsymbol{\lambda}+\frac{\partial \boldsymbol{\lambda}}{\partial \mathbf{p}} \delta \mathbf{p}\right)+\mathbf{M} \cdot \operatorname{curl}\left(\delta \boldsymbol{\lambda}+\frac{\partial \boldsymbol{\lambda}}{\partial \mathbf{p}} \delta \mathbf{p}\right)\right] d \Omega \\
& +\int_{\Omega}\left[\left(-\frac{\partial v}{\partial \mathbf{p}}\right) \operatorname{curl} \mathbf{A} \cdot \operatorname{curl} \boldsymbol{\lambda}+\frac{\partial \mathbf{J}}{\partial \mathbf{p}} \cdot \boldsymbol{\lambda}+\frac{\partial \mathbf{M}}{\partial \mathbf{p}} \cdot \operatorname{curl} \boldsymbol{\lambda}\right] \delta \mathbf{p} d \Omega
\end{aligned}
$$

where $\mathbf{g}_{\mathrm{A}} \equiv \partial \mathrm{g} / \partial \mathbf{A}$ and $\mathbf{g}_{\text {curlA }} \equiv \partial \mathrm{g} / \partial($ curlA $)$ correspond to the pseudo-sources of the adjoint system. For the simplicity of (3), we can replace arbitrary variables, $(\delta \mathbf{A}+(\partial \mathbf{A} / \partial \mathbf{p}) \delta \mathbf{p})$ and $(\delta \boldsymbol{\lambda}+(\partial \boldsymbol{\lambda} / \partial \mathbf{p}) \delta \mathbf{p})$, by an alternative notation, say $\overline{\boldsymbol{\lambda}}$ and $\overline{\mathbf{A}}$, respectively. Then, the sum of the three integrands, including the term $\overline{\mathbf{A}}$ on the right-hand side of (3), becomes zero because it represents the variational of the primary system. The rest, which is relevant to $\bar{\lambda}$, can be also set to zero as

$$
\begin{aligned}
-\int_{\Omega} v \operatorname{curl} \overline{\boldsymbol{\lambda}} \cdot \operatorname{curl} \boldsymbol{\lambda} d \Omega & \\
& +\int_{\Omega}\left[\mathrm{g}_{\mathbf{A}} \cdot \overline{\boldsymbol{\lambda}}+\mathbf{g}_{\operatorname{curl} \mathbf{A}} \cdot \operatorname{curl} \bar{\lambda}\right] d \Omega=0 .
\end{aligned}
$$

Equation (4) is the variational equation of the adjoint system and the pseudo-sources, $g_{A}$ and $g_{\text {curlA }}$, induced by the partial differential of the objective function, play the roles of the magnetic sources such as current density and permanent magnetization, respectively [4].

Therefore, a unified sensitivity formula applicable to TO of magnetostatic problems is given by

$$
\begin{aligned}
\frac{d \bar{F}}{d \mathbf{p}}=\int_{\Omega}\left[\left(-\frac{\partial v}{\partial \mathbf{p}}\right) \operatorname{curl} \mathbf{A} \cdot \operatorname{curl} \boldsymbol{\lambda}\right. & +\frac{\partial \mathbf{J}}{\partial \mathbf{p}} \cdot \boldsymbol{\lambda} \\
& \left.+\frac{\partial \mathbf{M}}{\partial \mathbf{p}} \cdot \operatorname{curl} \boldsymbol{\lambda}\right] d \Omega
\end{aligned}
$$

where the three integrands are the sensitivity coefficients with respect to system parameters $\mu, \mathbf{J}$ and $\mathbf{M}$, respectively.

\section{Distinction BetweEn OMD AND OSD}

After solving the dual formulation, consisting of the primary and the adjoint systems, the design sensitivity is easily obtained from (5). However, a distinction should be made between OMD and OSD type of problems. In order to explain the nature of this difference, the mathematical expressions and effects of material properties on the adjoint field distribution have been investigated.

First, compare the sensitivity coefficients of OMD and OSD in (5) with each other. The coefficient of OMD results from multiplying the primary field $\mathbf{A}$ and the adjoint field $\lambda$ together, whereas that of OSD just relates to $\lambda$. This implies that OMD

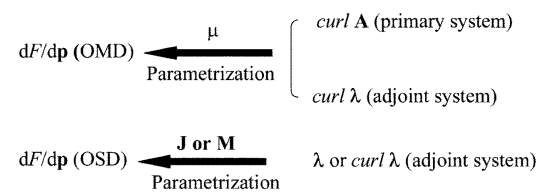

Fig. 1. Relationship between OMD, OSM, and dual system fields.

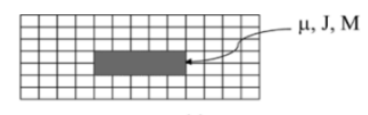

(a)

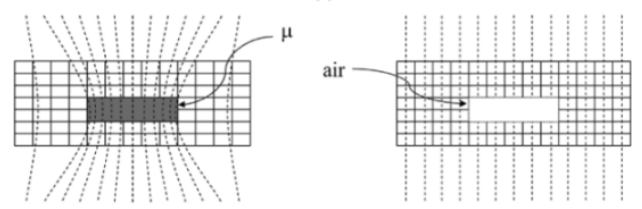

(b)

(c)

Fig. 2. Effects of material properties on the adjoint field distributions showing differences between OMD and OSM. (a) Presumed material distribution in the primary system. (b) Adjoint system of OMD. (c) Adjoint system of OSD.

is more strongly coupled with the fields of the dual system than OSD. Fig. 1 depicts the relation between OMD, OSD and the dual system fields.

By examining the effects of material properties on the adjoint fields, the meaning of the relationships shown in Fig. 1 can be clarified. Fig. 2 illustrates such effects of the materials on the adjoint fields. It is assumed that, in the primary system, the materials occupy gray cells of rectangular design domains at a certain design stage as in Fig. 2(a). The presence of the materials may contribute to the construction of the adjoint system and affect the fields generated by the pseudo-sources, or not. This depends only on the type of the design problem considered as illustrated by fields in Fig. 2(b) and (c).

In the case of OMD, the permeability is still present in the adjoint system, as well as in the primary one, and, thus, the fields of both systems are strongly distorted. That causes the increase of sensitivity coefficients over the cells already occupied by materials. If the values of permeability in the gray cells shown in Fig. 2(b) are greater by an order of magnitude than those of the adjacent cells, the material layout cannot change any more. In this case, the magnitude of the objective function that indicates how far the current design is from the optimum is not exactly reflected in the design sensitivity. This means that an abrupt change of the permeability is liable to result in OMD being trapped in local minima. This is the main reason why it is essential to the OMD image process that the material is forced to vary gradually from a void to solid state.

On the other hand, the current density and permanent magnetization of OSD are replaced in the adjoint system by air regions as shown in Fig. 2(c). Thus, the adjoint field distribution depends not on the relative value of distributed materials but on the magnitude and location of the objective function evaluated. This means that OSD itself, unlike OMD, does not have lots of local minima in the design space. Moreover, even if there are abrupt changes of material values assigned to design cells, OSD can easily converge to an optimum solution. These properties form the basis of a novel material updating algorithm for OSD, which allows each design cell to have only one state, that of a void or a solid. 


\section{NOVEL MATERIAL UPDATING SCHEME}

According to the conventional density method, design variables can be represented as

$$
\mathbf{J}(x, y)=\mathbf{J}_{s} p^{n}, \mathbf{M}(x, y)=\mathbf{M}_{s} p^{n}
$$

where, as usual, $p$ is the normalized design variable $(0 \leq p \leq 1)$ and $n$ is the penalization factor $(n>1)$. However, as already suggested in Section III, $p$ may be forced to be only a void (0) or a solid (1) and $n$ is set to 1 in the overall design procedure. As a result, the scheme does not require the somewhat complicated constraint penalty terms in the material parameterization as well as in the augmented objective function, which would enforce the intermediate values of design variables to be either 0 or 1 .

Assuming the desired volume $V_{o}$ of the whole design space to be initially given, the proposed iterative design process involves the following steps.

1) Solve the primary and adjoint systems, successively.

2) Compute design sensitivity using the analytical formula (5).

3) Calculate a mutation factor $\gamma_{i+1} \equiv \gamma_{\max } \times 0.85^{i}$ where $\gamma_{\max }$ is the maximum value of the amount of topological change in the next design stage with respect to the current layout.

Inner loop: $\{$.

Search $\alpha$ to satisfy $V_{i+1} / V_{o} \leq \varepsilon_{1}$ and $\left(V_{i+1}-\right.$ $\left.V_{o}\right) / V_{o} \times 100 \leq \varepsilon_{2} \times \gamma_{i+1}$ where $V_{i+1}$ is decided according to transient values of $\alpha$ and its corresponding material sensitivity $P_{\text {temp }}=P_{i}+\alpha \Delta P$. $\}$.

Accumulate material sensitivity $P\left(P_{i+1}=\right.$ $\left.P_{i}+\alpha \Delta P\right)$.

4) Enforce each design cell $(p)$ to be 0 or 1 .

5) Repeat the above procedure until the objective function converges.

\section{EXAMPLES}

In a previous paper [4], the authors presented a program architecture that allowed incorporation of the continuum design sensitivity analysis (CDSA) into existing EM software packages without the need to modify the source code. In this paper, after slight modifications to a part of the optimization module and the command file, the same program architecture has been applied to OSD problems. The design domain to be occupied by materials should be subdivided into multiple individual regions so that material properties can be imposed in each region defined prior to FE mesh generation. The individual regions correspond to design cells and a linear static OPERA-2D solver was used for analysis.

\section{A. Optimized Current Distribution}

The algorithm has been applied to a MRI test device with magnetic iron shield [1], [3] (refer to Fig. 5). The design goal is to find an optimal configuration of coils, carrying a current of 1 A, which achieves uniform field distribution at 9 measuring points. The objective function is given as

$$
F=\sum_{i=1}^{9}\left(H_{\mathrm{yi}}-H_{\mathrm{yo}}\right)^{2}
$$

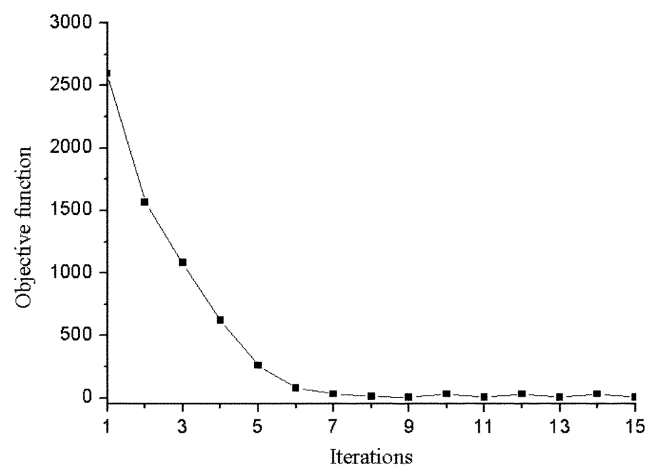

Fig. 3. Convergence of the objective function versus iterations.

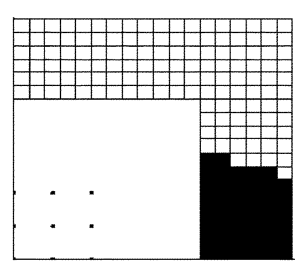

(a)

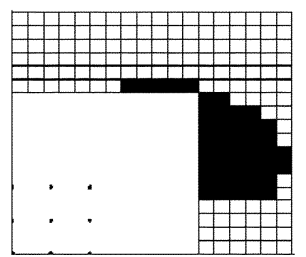

(c)

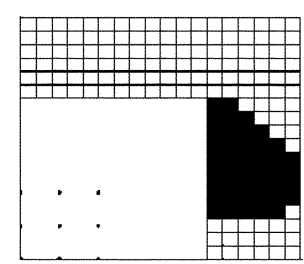

(b)

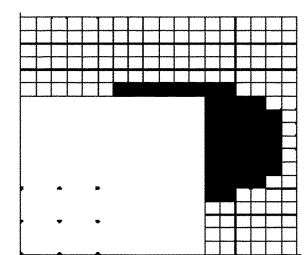

(d)
Fig. 4. Changes of current distribution during optimization. (a) One iteration. (b) Three iterations. (c) Five iterations. (d) Nine iterations.

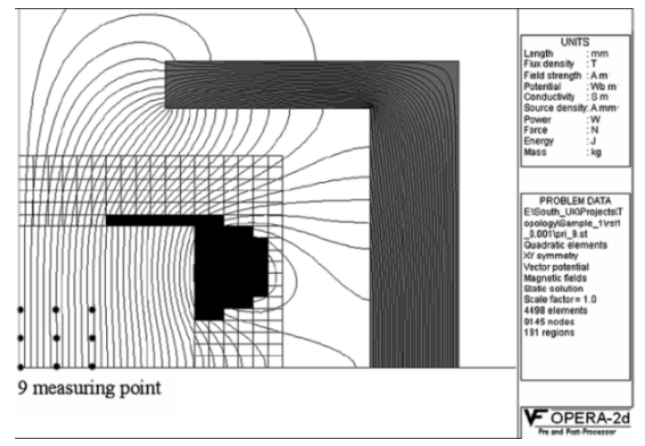

Fig. 5. Flux distribution after optimization.

where $H_{\mathrm{yi}}$ and $H_{\mathrm{yo}}$ are the $y$-component of the magnetic field intensity computed at the $i$ th measuring point and the desired constant value of $36.26 \mathrm{~A} / \mathrm{m}$, respectively. The algorithm was executed under initial conditions of $V_{o}=24 \%$ of all design cells and $\gamma_{\max }=35 \%$.

After only nine iterations, field intensities within $2 \%$ of the desired value have been obtained as demonstrated in Fig. 3. Fig. 4 gives examples of current distributions during optimization, whereas the flux pattern after optimization is shown in Fig. 5. It is known that other existing material updating schemes usually require more than 100 iterations to obtain the optimized coil and flux distributions presented here. 


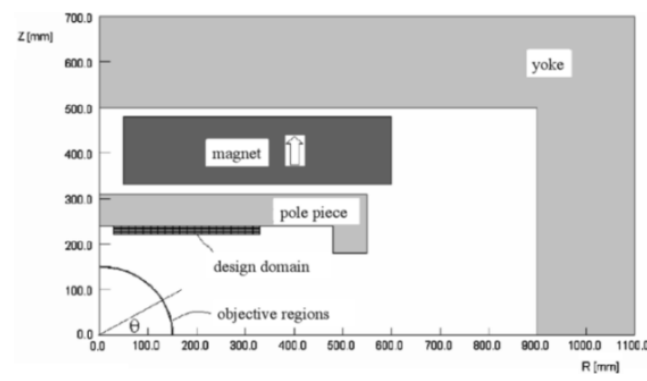

Fig. 6. Quarter model of an open permanent magnet-type MRI.

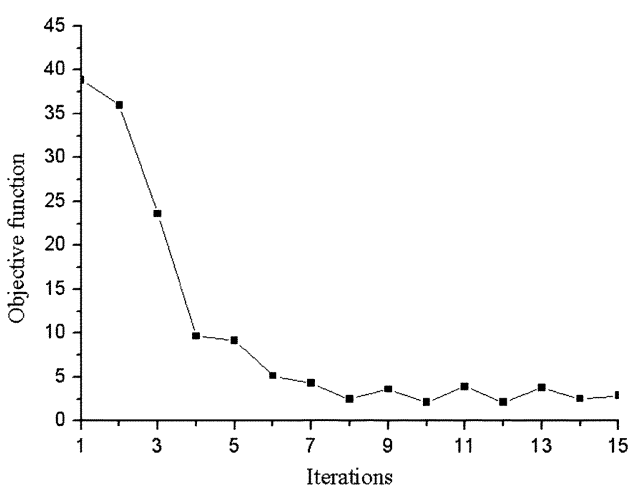

Fig. 7. Convergence of the objective function versus iterations.

\section{B. Optimized Permanent Magnet Distribution}

Fig. 6 shows a quarter of a model of a permanent magnet assembly for an MRI device where the residual flux density of the magnet is $1.21 \mathrm{~T}$ [5]. Although the actual assembly is three dimensional, as it has two columns, here it has been simplified to an axi-symmetric problem. The design goal is to find an optimized distribution of shimming magnets over the pole piece surface to obtain homogenous field distribution in a $30-\mathrm{cm}$ diameter spherical volume (DSV). The shimming magnet has the residual flux density of $0.22 \mathrm{~T}$ and thickness of $3 \mathrm{~mm}$ and the domain is subdivided into 120 separate regions.

The objective function and design variable are defined as

$$
F=\sum_{i=1}^{45}\left(H_{\mathrm{zi}}-H_{\mathrm{zo}}\right)^{2}, \quad \mathbf{M}(x, y)=\mathbf{M}_{s}(P) p^{n}
$$

where $H_{\mathrm{zi}}$ is the $z$-component of the magnetic field intensity computed over the objective regions (consisting of 45 individual quadrilateral regions along a $90^{\circ}$ arc at a $300-\mathrm{mm}$ radius), and $H_{\text {zo }}$ is the desired value. In (6), $\mathbf{M}_{s}(P)$ in each cell is forced to have $\pm \mathbf{M}_{s}$ according to the sign of the accumulated design sensitivity $P$ during optimization in order to take into account the direction of $\mathbf{M}_{s}$. The algorithm was executed under initial conditions of $V_{o}=42 \%$ of all design cells and $\gamma_{\max }=20 \%$.

The convergence of the objective function and the shimming magnets distribution during the optimization are shown in Figs. 7 and 8, respectively. Fig. 9 compares the $z$ component of magnetic fields over the surface of the DSV before and after optimization, where the uniformity of the fields is improved four times compared with the initial design.

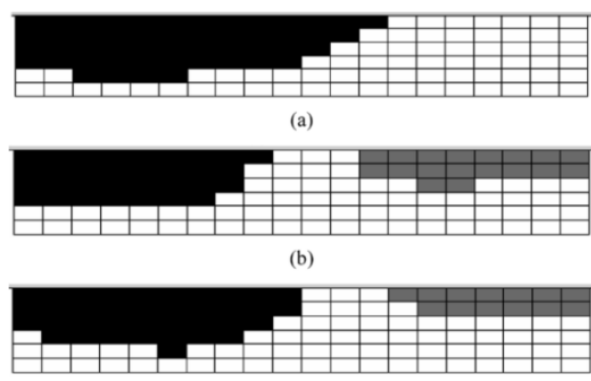

(c)

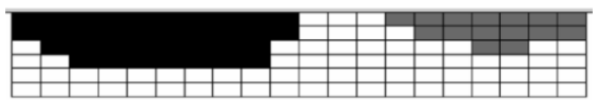

(d)

Fig. 8. Changes of shimming magnet distribution during optimization. Black cell: $+\mathbf{M}_{\mathrm{s}}$. Gray cell: $-\mathbf{M}_{\mathrm{s}}$ ). (a) One iteration. (b) Three iterations. (c) Seven iterations. (d) Ten iterations.

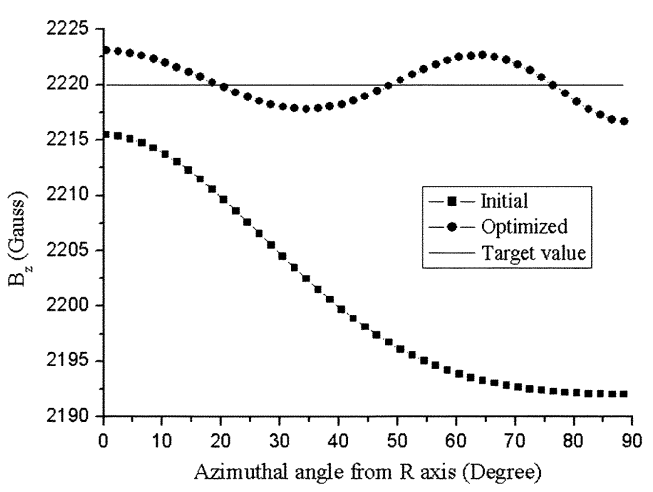

Fig. 9. Comparison of field distributions before and after optimization.

\section{CONCLUSION}

This paper presents a new and efficient approach to optimized source distribution problems by emphasizing differences in relation to optimized material distribution formulations. The proposed material updating scheme, combined with commercial finite element software, offers advantages of easy implementation and fast convergence. The results demonstrate that optimized magnetic source distributions can be found without the need for invalid material states between a void and a solid that lead to complicated implementations and unnecessarily lengthy iterations.

\section{REFERENCES}

[1] D. N. Dyck et al., "A method of computing the sensitivity of electromagnetic quantities to changes in material and sources," IEEE Trans. Magn., vol. 30, no. 5, pp. 3415-3418, Sep. 1994.

[2] J.-K. Byun et al., "Topology optimization of electrical devices using mutual energy and sensitivity," IEEE Trans. Magn., vol. 35, no. 5, pp. 3718-3720, Sep. 1999.

[3] J.-H. Lee et al., "Faster calculation of sensitivity in the source current distribution problem using reciprocity theorem," IEEE Trans. Magn., vol. 37, no. 5, pp. 3596-3599, Sep. 2001.

[4] D.-H. Kim et al., "Applying continuum design sensitivity analysis combined with standard EM software to shape optimization in magnetostatic problems," IEEE Trans. Magn., vol. 40, no. 2, pp. 1156-1159, Mar. 2004.

[5] D.-H. Kim et al., "3-D optimal shape design of ferromagnetic pole in MRI magnet of open permanent-magnet type," IEEE Trans. Appl. Supercond., vol. 12, no. 1, pp. 1467-1470, Mar. 2002. 